\title{
Molecular epidemiology of trachoma in a Gambian village
}

\author{
R L Bailey, L Hayes, M Pickett, H C Whittle, M E Ward, D C W Mabey
}

\begin{abstract}
The application of a diagnostic and genotyping technique based on the polymerase chain reaction (PCR) to the study of trachoma epidemiology in the Gambian village of Jali is reported. PCR based on the major outer membrane protein (MOMP) gene of Chlamydia trachomatis appears to be more sensitive than either isolation or antigen detection by enzyme immunoassay; it had a specificity of $95 \%$ and sensitivity of $51 \%$ against clinical signs. PCR genotyping identified genotypes $A$ and $B$ of Chlamydia trachomatis circulating in Jali. Sequencing revealed a Pstl restriction endonuclease site in the amplified MOMP gene of some B strains but not others; Pstl digestion of the PCR product proved an easy method of distinguishing these strains. The distribution of serotypes and B strain variants shows a significant degree of household clustering $(p<0.001)$. PCR based genotyping combined with strain typing provides a new and powerful epidemiological tool for the study of transmission events in trachoma.
\end{abstract}

(Brf Ophthalmol 1994; 78: 813-817)

Trachoma, the world's leading infectious cause of blindness, is estimated to afflict some 500 million people, of whom 7 million are blind. ${ }^{1}$ It is caused by serovars A, B, Ba, and C of Chlamydia trachomatis. In its early (active) stage, which is seen principally among children in endemic areas, it is characterised by a chronic follicular conjunctivitis which affects principally the upper subtarsal conjunctiva. Among older individuals in endemic areas, conjunctival scarring replaces the follicles and may lead to entropion, trichiasis, and hence blindness due to constant corneal abrasion. It has been suggested that repeated reinfection is a prerequisite for these blinding sequelae. $^{2}$

The prevention of blindness due to trachoma depends on a reduction in the transmission of $C$ trachomatis from eye to eye in communities where the disease is endemic. We and others have shown that cases of trachoma tend to cluster by family and by household, implying that transmission occurs within the family. ${ }^{34}$ Microbiological techniques for the detection of the organism are an essential adjunct to clinical examination in our attempts to understand the epidemiology of trachoma and hence to formulate rational control measures. Techniques whereby strains can be distinguished from one another - for example, by serotyping, provide a more incisive epidemiological tool for this purpose.

Methods previously used to diagnose ocular chlamydial infection in trachoma include direct smears stained with iodine or Giemsa, isolation in eggs or tissue culture, antigen detection by direct immunofluorescence or enzyme immunoassay (EIA), and DNA hybridisation. None of these methods is sufficiently sensitive; typically none is positive in more than $50 \%$ of clinical cases of active trachoma. ${ }^{5-7}$

Recently the polymerase chain reaction (PCR) has been used to amplify chlamydial DNA in clinical specimens. This technique is sensitive and specific for the diagnosis of genital chlamydial infections, ${ }^{8-10}$ and a study from Tanzania has found it to be more sensitive than other techniques for the diagnosis of ocular infection in trachoma. ${ }^{11}$ However in the latter study, $24 \%$ of individuals with no clinical signs of trachoma were identified as infected with $C$ trachomatis by PCR, suggesting either that there was an extremely high level of clinically inapparent infection or, alternatively, that the increased sensitivity of their PCR was achieved at the expense of specificity.

We report here a study from a Gambian village in which trachoma is endemic, in which amplification of the chlamydial major outer membrane protein (MOMP) gene by PCR has been used for the diagnosis of ocular chlamydial infection. We also report on the distribution of chlamydial genotypes within the village, as determined by a novel method based on the use of type specific primers in the PCR. Within genotype strain variants identified by sequencing have led to a straightforward method of subtyping B strains. The use and significance of this are discussed.

\section{Patients and methods}

STUDY SUBJECTS AND SPECIMEN COLLECTION The Gambian village of Jali has been described previously. ${ }^{3}$ It contains approximately 950 inhabitants, all Muslims of the Mandinka tribe and for the most part subsistence farmers, living in 48 compounds, or household units based on the extended family.

In October 1990 the eyes of all inhabitants were examined by a trained observer (RLB) using a $\times 2$ binocular loupe (Dixey Ltd) and pen torch. After eversion of the eyelids, trachoma was graded by the appearance of the subtarsal conjunctiva according to the criteria of Dawson et $a l^{1}$ as absent or insignificant (T0), mild (T1), moderate (T2), or severe (T3), according to the number of follicles and the degree of papillary hypertrophy. The presence or absence of scarring sequelae was also recorded.

Swabs were taken from the upper subtarsal conjunctiva and tested for chlamydial DNA by PCR and for chlamydial antigen by amplified enzyme immunoassay (IDEIA Chlamydia, Novo 
Biolabs, UK). Subjects tested included all persons with clinical disease $(n=96)$ and a proportion of those who were clinically negative $(n=37)$. The clinically negative subjects were selected from two households, one containing substantial numbers of trachoma cases, the other free of clinical trachoma; all members of these households were sampled.

Swabs were immediately placed in $1 \mathrm{ml}$ of IDEIA transport medium supplied by the manufacturer. These specimens were stored at $4^{\circ} \mathrm{C}$ for up to 6 hours, then frozen at $-20^{\circ} \mathrm{C}$ for up to 4 months before being assayed. A volume of $200 \mu \mathrm{l}$ of transport medium from each swab was aliquoted and $10 \mu \mathrm{l}$ of this was used for DNA amplification by the PCR. Aliquots were taken using strict sterile technique in a microbiological safety cabinet.

\section{ETHICAL CONSIDERATIONS}

This study was approved by the joint Medical Research Council/Gambia Government ethics committee.

\section{ANTIGEN DETECTION}

Chlamydia antigen was assayed using the IDEIA Chlamydia kit (Novo Biolabs) in accordance with instructions supplied by the manufacturer. This assay detects genus specific chlamydial lipopolysaccharide antigen.

\section{PCR AMPLIFICATION OF MOMP GENES AND PCR SEROTYPING}

Nucleic acids were prepared from the swabs in enzyme linked immunosorbent assay (ELISA) transport medium as previously described ${ }^{12} ; 5 \mu \mathrm{l}$ of each DNA preparation was used in the first PCR.

PCR amplification of chlamydial DNA from

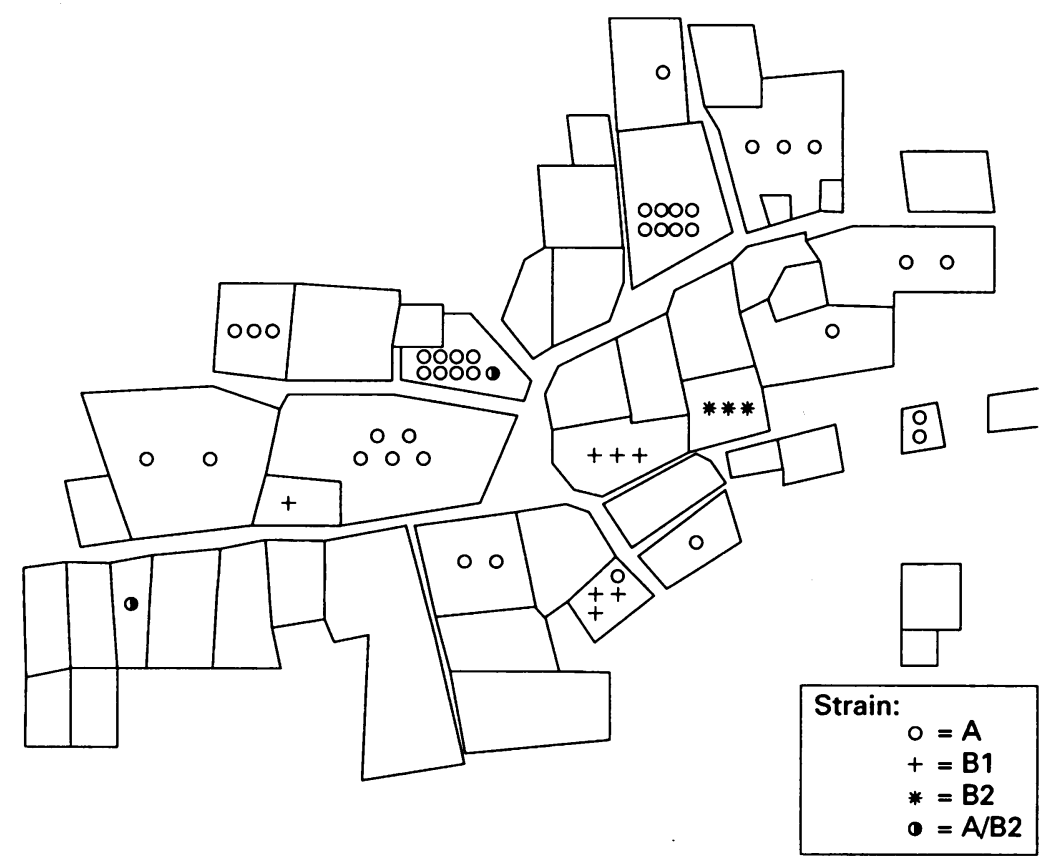

Figure 1 Map of Fali village, showing genotype and strain type distribution by household in October 1990. The lines on the map represent the fences which make up household boundaries. the eye swabs was performed on a Perkin Elmer thermal cycler in two stages as fully described elsewhere. ${ }^{12}$ The first stage amplified almost the whole MOMP gene, while the second stage amplified serovar specific products of characteristic molecular weights. A $5 \mu \mathrm{l}$ sample of each amplified product was examined by agarose gel electrophoresis and the serovars of infecting strains were determined from the characteristic sizes of the second stage PCR products. At least two negative controls were run on each gel and in all cases showed negative.

\section{RESTRICTION DIGESTS}

Samples of $10 \mu$ l of second stage PCR product were incubated with $1 \mu \mathrm{l}$ of Pstl (Stratagene) at $37^{\circ} \mathrm{C}$ for 1 hour. The products were analysed by agarose gel electrophoresis.

\section{SEQUENCING}

Biotinylated top and bottom strands were generated by PCR as previously described. ${ }^{12}$ The primers selectively amplified portions of the A and $B$ MOMP genes. The strands were bound to magnetic streptavidin coated beads (Dynabeads M-280; Dynal, Oslo, Norway) and sequenced by the dideoxy chain termination method using primers based on the known gene sequences.

\section{STATISTICAL ANALYSIS}

The distribution of serotypes to households among the PCR positive cases would be amenable to a $\chi^{2}$ test if the cell sizes were not so small. In this case the distributional assumptions were invalid and therefore significance was tested by 1000 simulations in which the serovar B cases were modelled to occur at random among the PCR positive cases with a probability equal to the observed proportion. This was carried out on a Toshiba portable computer using the 'ranbin' function in SAS software.

The significance of the distribution of serovar $B$ cases to households was tested by computing a $\chi^{2}$ statistic for each simulation and estimated as the proportion of the 1000 having a $\chi^{2}$ value more extreme than that computed from the observed distribution. ${ }^{3}$

\section{Results}

CLINICAL EXAMINATION

A total of 844 subjects were examined; 96 $(11 \cdot 3 \%)$ were graded as clinically active cases of trachoma. Of these, 56 were graded as mild (T1), 28 as moderate (T2), and 12 as severe (T3).

\section{ANTIGEN DETECTION}

Twenty four (25\%) swabs taken from clinically active individuals were positive for chlamydial antigen. None of 37 swabs taken from clinical negatives was antigen positive.

For severe disease (T3) 10 of 12 were antigen positive ( $83 \%$ ); for moderate disease (T2) eight of $28(29 \%)$, and for mild disease (T1) six of 56 (11\%). Thus the antigen detection ELISA had a 


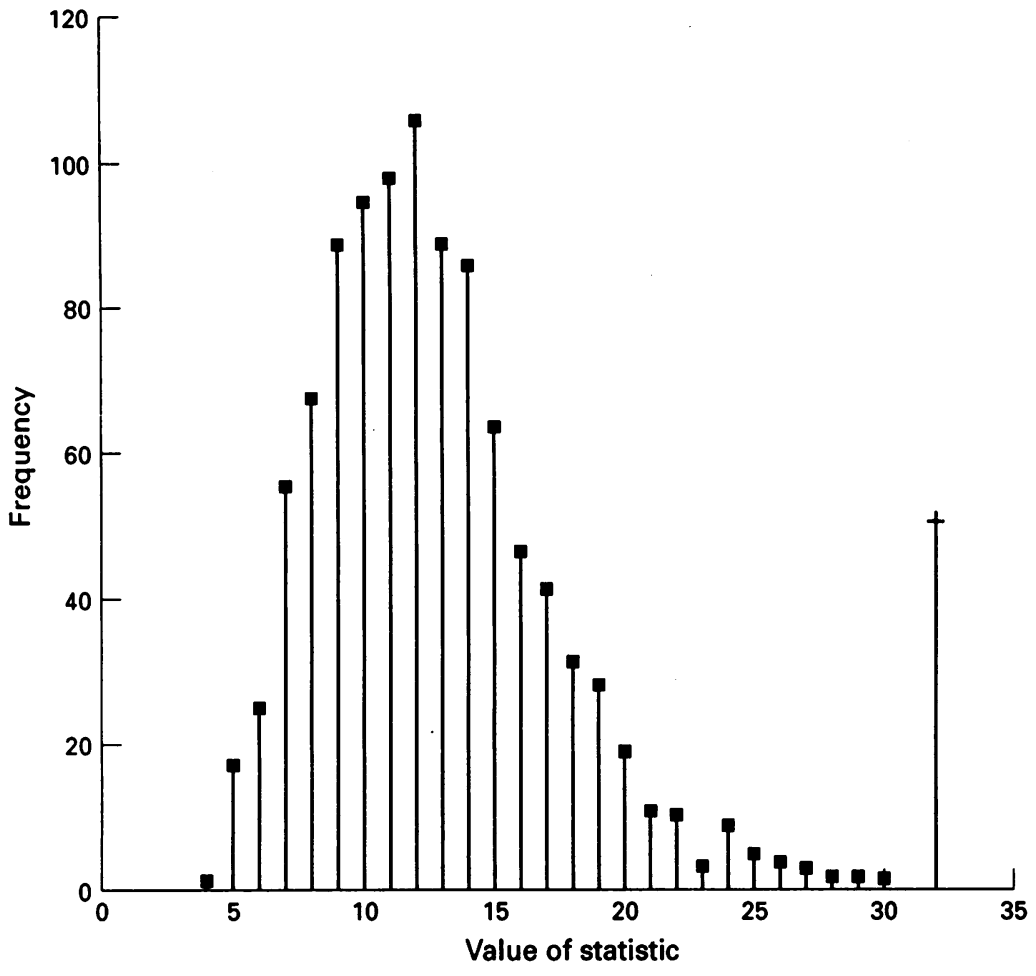

Figure 2 Result of 1000 simulations of the null hypothesis that serotypes $A$ and $B$ are randomly distributed among households. The observed value of the statistic (32.03) lies outside all 1000 simulations suggesting $p<0.001$.

specificity of $100 \%$ and a sensitivity of $25 \%$ against clinical signs.

\section{DIAGNOSIS BY PCR}

Of the 96 subjects with clinical trachoma 49 were positive by PCR (51\%). Of 37 clinical negatives two were positive by PCR (5\%). Neither of these individuals had normal eyes; one had evidence of both inflammation and a moderate degree of conjunctival scarring; the other had follicles on both upper tarsal conjunctivae, but less than the five required for the accepted diagnosis of active trachoma. Both these subjects lived in the household where disease was present. For mild active disease (T1) 25 out of 56 samples were positive (45\%); for moderate disease (T2) 16 out of 28 (57\%); and for severe disease (T3) eight out of 12 (67\%) were positive. Against clinical signs the PCR thus had a diagnostic specificity of $95 \%$ and a sensitivity of $51 \%$.

\section{GENOTYPING BY PCR}

The genotypes identified in Jali by the PCR method were A and B; no genotype C DNA was detected. Genotype A was detected in 41/51 $(80 \%)$ and genotype B in $12 / 51(24 \%)$ positive samples. Two individuals were infected with both types $A$ and $B$.

$C$ trachomatis MOMP gene DNA was detected in eye swabs from 51 individuals in 15 households. In 10 households only serotype $A$ was detected, in three households only type B was detected, and in two households both serotypes were detected. The detection of genotypes $A$ and $B$ together in two swabs was confirmed by sequencing MOMP variable domains 1 and 2 of both serovars from those individuals.
STRAIN TYPING

Sequencing of MOMP variable domain 1 from individuals infected with serotype $B$ showed that two strains of B were present which differed by a point mutation resulting in the creation of a Pstl restriction endonuclease cleavage site in one of the strains.

Pstl digests of second stage genotypes B PCR products demonstrated this strain in five of the 12 individuals positive for genotype B. One sample gave too low a signal for the strain type to be determined.

The household distribution of genotypes and of $B$ genotype variants is shown in Figure 1 .

\section{STATISTICAL ANALYSIS}

The distribution of the $\chi^{2}$ statistics for the 1000 simulations of the household distribution of genotypes A and B is shown in Figure 2; the observed value is more extreme than all of the simulations implying that the genotypes are clustered by household with $\mathrm{p}<0 \cdot 001$. The type $B$ strain variant distribution supports this idea; within the clusters of three individuals infected with this genotype all were of the same strain.

\section{Discussion}

A number of studies have recently been published in which the detection of chlamydial DNA following amplification by PCR has been compared with conventional techniques for the diagnosis of genital chlamydial infections. Chlamydial sequences selected for amplification have included the MOMP gene, the cryptic plasmid, and the 16S ribosomal RNA gene. ${ }^{8-10}$ PCR has been shown to be extremely sensitive in these studies but less than $100 \%$ specific when compared with culture or antigen detection. This apparent lack of specificity may be due to the poor sensitivity of the 'standard'; it is generally agreed that culture even in optimal circumstances is less than $100 \%$ sensitive.

In trachoma, in contrast with genital chlamydial infection, there are generally accepted clinical criteria for the identification of infected individuals. ${ }^{13}$ However, numerous studies have shown that none of the existing methods is able to detect Chlamydia in all subjects with clinical signs of active disease. ${ }^{\text {5-7 }}$ In a previous study in Jali village we were only able to isolate $C$ trachomatis from the eyes of $12 \cdot 4 \%$, $33.3 \%$, and $36.8 \%$ of subjects with mild, moderate, and severe active trachoma respectively. ${ }^{5}$ Schachter et al, using isolation, DNA hybridisation, and antigen detection by EIA and by immunofluorescence were only able to demonstrate infection in $49 \%$ of 91 Egyptian children with moderate to severe inflammatory trachoma. ${ }^{6}$ There is clearly a need for a more sensitive diagnostic method.

Studies in the cynomolgus monkey model of trachoma have shown that PCR is more sensitive than isolation or antigen detection for the diagnosis of ocular chlamydial infection. ${ }^{14}$ Bobo et al have recently used PCR for the detection of chlamydial DNA in ocular swabs from 234 Tanzanian children in a trachoma endemic community. They detected chlamydial DNA in 54 of 
100 children with mild to moderate active disease (TF) and 35 of 37 with severe disease. ${ }^{11}$ However, they also detected chlamydial DNA in 23 of 97 children with no clinical signs of trachoma.

Many studies in trachoma endemic regions have found a small number of subjects without clinical trachoma in whom $C$ trachomatis can be detected by isolation, but this has generally been less than $10 \%^{5715}$; it may be that the higher percentage found in the Tanzanian study reflects a different epidemiological situation from that in our Gambian community, with a greater 'pressure of infection' resulting in a higher proportion of Chlamydia infected subjects with normal eyes. PCR is susceptible to false positive reactions owing to its sensitivity to contamination by extraneous DNA, but may also pick up a higher proportion of genuine positives without clinical signs (such as those incubating disease) than the other available methods.

In the present study we have confirmed that PCR is more sensitive than isolation or antigen detection for the diagnosis of ocular chlamydial infection in trachoma, particularly in those with mild to moderate disease. In our study the specificity of PCR against clinical signs was $95 \%$ (cf $76 \%$ found by Bobo et al ${ }^{11}$ ).

The role of cryptic chlamydial infection in the pathogenesis of trachomatous conjunctival scarring remains unclear. Most subjects with scarring in endemic communities are chlamydial isolation negative, but chlamydial antigen can be detected in a proportion of them. ${ }^{5}$ is It would be of great interest to use the more sensitive PCR technique to look for evidence of chlamydial infection in subjects with conjunctival scarring.

Previous studies in Iran, Saudi Arabia, Taiwan, and Tanzania have investigated the distribution of chlamydial serotypes in trachoma endemic communities. ${ }^{216-19} \mathrm{~A}$ combination of two techniques was used; isolates were typed with adsorbed polyclonal antisera and the type specificity of serum and/or tear antibodies were tested against standard chlamydial serotypes. Clearly the type specificity of the antibody response may be difficult to interpret, since it may reflect past or current infection. The conclusion of these studies was similar to our own - namely, that in most households only a single serotype is present, and here we have formally shown, using Monte Carlo methods, that this is unlikely to have occurred by chance.

These findings confirm the hypothesis that infection in trachoma is mainly transmitted within the household. In a cross sectional study, they do not allow us to distinguish between persistent infection and repeated reinfection from other household members. Thus, Nichols et al concluded from their studies in Saudi Arabia that persistent infection was the cause of trachoma, whereas Grayston et al concluded from their studies in Taiwan that repeated reinfection was a prerequisite. ${ }^{218}$ The potential to distinguish strain variants by molecular methods in longitudinal studies is likely to determine which interpretation is correct.

The use of PCR allows infections to be typed without the need for isolation. This is a major advantage for field studies where it may be difficult or impossible to maintain a cold chain. Moreover, RFLP analysis or sequencing of amplified MOMP gene sequences can lead to the identification of within serotype variants, thereby providing finer specificity for epidemiological studies of transmission than can be obtained by serotyping alone. ${ }^{20}$ Preliminary studies in Jali village have also identified three variants of serotype A caused by point mutations in the MOMP gene. ${ }^{12}$ Unlike the B variants these are not separable by restriction fragment length polymorphism but may be separable by single stranded conformational polymorphism; this is under investigation at present. Longitudinal studies in which the transmission of these variants within the village is investigated are likely to improve our understanding of the epidemiology and pathogenesis of trachoma, and hence to contribute to the design of effective intervention strategies against this major blinding disease.

We gratefully acknowledge the financial support of the Edna McConnell Clark Foundation (grants 13390 to DM and 13290 to $M W)$ and of the MRC (training fellowship to RB). We are grateful to Dr B M Greenwood, director of the Medical Research Council Laboratories in Gambia, for his support; to Dr R Whitehead and the staff of the Dunn Nutrition Field station, Keneba for the provision of field facilities; to Hassan Joof for field work, and to Mariama Sanneh for technical assistance. This study would not have been possible but for the good humoured cooperation of the people of Jali, to whom we are also grateful.

1 Dawson CR, Jones BR, Tarizzo ML. Guide to trachoma control in programmes for the prevention of blindness. Geneva: World Health Organisation, 1981.

2 Grayston JT, Wang S-P, Yeh L-J, Kuo C-C. Importance of reinfection in the pathogenesis of trachoma. Rev Infect Dis reinfection in the

3 Bailey R, Osmond C, Mabey DCW, Whittle HC, Ward ME. Analysis of the household distribution of trachoma in a Gambian village using a Monte Carlo simulation procedure. Int f Epidemiol 1989; 18: 944-51.

4 Barenfanger J. Studies on the role of the family unit in the transmission of trachoma. Am $\mathcal{F}$ Trop Med Hyg 1975; 24: 509-15.

5 Mabey DCW, Robertson JN, Ward ME. Detection of Chlamydia trachomatis by enzyme immunoassay in patients with trachoma. Lancet 1987; ii: 1491-2.

6 Schachter J, Moncada J, Dawson CR, Sheppard J, Courtright $\mathrm{P}$, Said ME, et al. Nonculture methods for diagnosing
chlamydial infection in patients with trachoma: a clue to the pathogenesis of the disease? $\mathcal{Y}$ Infect Dis 1988; 158: 1347-52.

7 Taylor HR, Rapoza PA, West S, Johnson S, Munoz B, Katala $S$, et al. The epidemiology of infection in trachoma. Invest Ophthalmol Vis Sci 1989; 30: 1823-33.

8 Claas HC, Melchers WJ, de Bruijn IH, de Graaf M, van Dijk WC, Lindeman J, et al. Detection of Chlamydia trachomatis in clinical specimens by the polymerase chain reaction. Eur $\mathcal{F}$ Clin Microbiol Infect Dis 1990; 9: 864-8.

9 Ostergaard L, Birkelund S, Christiansen G. Use of polymerase chain reaction for detection of Chlamydia trachomatis. $\mathcal{F}$ Clin Microbiol 1990; 28: 1254-60.

10 Bobo L, Coutlee F, Yolken RH, Quinn T, Viscidi RP. Diagnosis of Chlamydia trachomatis cervical infection by detection of amplified DNA with an enzyme immunoassay. detection of amplified DNA with an

11 Bobo L, Munoz B, Viscidi R, Quinn T, Mkocha H, West S. Diagnosis of Chlamydia trachomatis eye infection in Tanzania by polymerase chain reaction/enzyme immunoassay. Lancet 1991; 338: 847-50.

12 Hayes LJ, Bailey RL, Mabey DCW, Clarke IN, Pickett MA, Watt PJ, et al. Genotyping of Chlamydia trachomatis from a trachoma-endemic village in the Gambia by a nested polymerase chain reaction: identification of strain variants. f Infect Dis 1992; 166: 1173-7.

13 Thylefors B, Dawson CR, Jones BR, West SK, Taylor HR. A simple system for the assessment of trachoma and its simple system for the assessment of trach
complications. Bull WHO 1987; 65: 477-83.

14 Holland SM, Hudson AP, Bobo L, Whittum-Hudson JA Viscidi RP, Quinn TC, et al. Demonstration of chlamydial RNA and DNA during a culture negative state. Infect Immun RNA and DNA during

15 Ward M, Bailey R, Lesley A, Kajbaf M, Robertson J, Mabey D. Persistent inapparent chlamydial infection in a trachoma endemic community in The Gambia. Scand F Infect Dis 1990 (Suppl 69): 137-48.

16 Treharne JD. Seroepidemiological studies of trachoma. In: Mardh P-A, Holes KK, Oriel JD, Piot P, Schachter J, eds. Chlamydial infections. Amsterdam: Elsevier, 1982: 83-6.

17 Treharne JD. The microbial epidemiology of trachoma. Int Ophthalmol 1988; 12: 25-9.

18 Nichols RL, Von Fritzinger K, McComb DE. Epidemiological data derived from immunotyping of $\mathbf{3 3 8}$ trachoma strains isolated from children in Saudi Arabia. In: Nichols RL, ed. 
Trachoma and related disorders caused by chlamydial agents. Amsterdam: Excerpta Medica, 1971: 337-57.

19 Taylor HR, Siler JA, Mkocha HA, Munoz B, West S. The natural history of endemic trachoma: a longitudinal study. Am f Trop Med Hyg 1992; 46: 552-9.
20 Dean D, Schachter J, Dawson CR, Stephens RS. Comparison of the major outer membrane protein variant sequence regions of $\mathrm{B} / \mathrm{Ba}$ isolates: a molecular epidemiologic approach to Chlamydia trachomatis infections. F Infect Dis 1992; 166: 383-92. 